\title{
CRATERING OF A COMET NUCLEUS BY METEOROIDS
}

\author{
K. Jach ${ }^{1}$, L. Kortas ${ }^{2}$, J. Leliwa-Kopystyński ${ }^{3,4}$, M. Morka ${ }^{1}$, M. Mroczkowski ${ }^{1}$, R. Panowicz ${ }^{1}$, R. Świerczyński ${ }^{1}$, and \\ P. Wolański
}

${ }^{1}$ Military University of Technology, Kaliskiego 2, 00-908 Warszawa 49, Poland

${ }^{2}$ University of Mining and Metalurgy, Al. Mickiewicza 30, 30-059 Krakow, Poland

${ }^{3}$ University of Warsaw, Institute of Geophysics, Pasteura 7, 02-093 Warszawa, Poland

${ }^{4}$ Space Research Center of PAN, Bartycka 18A, 00-716 Warszawa, Poland

${ }^{5}$ Warsaw University of Technology, Institute of Heat Engineering, Nowowiejska 25/29, 00-665 Warszawa, Poland

\begin{abstract}
The two-dimensional axisymmetric hydrocode model of free particles is applied to the calculation of response of a comet nucleus to a meteorite impact. The nucleus is assumed to be spherical with a radius of $1 \mathrm{~km}$. It is composed of a porous granular mixture of water ice and of mineral. Initial temperature is $50 \mathrm{~K}$. Porosity is $\psi=0.6$ and the mean density is $\rho=400 \mathrm{~kg} \mathrm{~m}^{-3}$. Impactor radius is equal to $1 \mathrm{~m}$ and its mean density is equal to that of the nucleus. Impact velocity is $10 \mathrm{~km} \mathrm{~s}^{-1}$. A normal impact is considered. Particular forms for the equations of state (EOS) for the real medium (water ice and rock) as well as for an artificial medium of very low-density $\left(40 \mathrm{~kg} \mathrm{~m}^{-3}\right)$ filling up the voids are used. The cohesion is included in the constitutive model of the constituents. Numerical modelling provides the time dependent fields of pressure, density, temperature, and particle velocity in the vicinity of an impact point. The evolution of the field of temperature correlated with the function for kinetics of amorphous to crystalline phase transition permits discussion of the impact-induced crystallization of presumably initially amorphous ice.
\end{abstract}

C1999 COSPAR. Published by Elsevier Science Ltd.

\section{MODEL OF COMET NUCLEUS}

There exists a large diversity in details concerning modeling of comet nuclei. However, the dominating abundance of water ice as well as the high porosity of nuclei are well established. Other features are more or less controversial. Among them is the state of the ice: amorphous or crystalline, see e.g. Rickman (1991) and Kauchi et al. (1994). The last authors concluded: "Icy grains which formed the Uranian and Neptunian satellites and comets were initially amorphous, if they were formed from the icy grains preserved from the molecular cloud stage". The form of ice depends on temperature of formation of a comet nucleus, on contents of radioactive nuclei including short lived $\mathrm{Al}^{26}$ (Prialnik et al. 1987), on history of a comet from formation epoch till the recent time, and on actual properties of comet's orbit, in particular on perihelion distances. Intrinsic radioactivity, if sufficiently intense, can heat a presumably amorphous ice and transform it to the crystalline form. Energy of solar radiation or accumulated energy of many impacts can transform ice from amorphous to crystalline form in a layer spreading downward from the surface level to a certain depth. Study of impact induced phase transition from amorphous to crystalline phase of water ice is one of the goals of this work. This phase transition driven by solar flux of energy incident on comet surface was recently studied by Podolak and Prialnik (1996).

We suppose that the grains of water ice and of mineral as well as the voids are randomly mixed. They form the nucleus that is statistically or "macroscopically" uniform, Figure 1. However, our two-dimensional (2D) geometry requires axial symmetry: the $z$-axis follows the direction of impactor movement and is perpendicular to the target; it is directed downward into the nucleus, with $z=0$ at the surface. In the 2D case the grains of ice and those of rock as 
well as the voids are of toroidal shape. For the purpose of nurnerical algorithm, each of the cross sections of a torus visible as small rectangles in Figure 1 must be divided into some number, in our case 9 of the material points. We need therefore the appropriate equations of state (EOS) and the constitutive equations (CE) to describe icy and mineral matter of a particular torus. Moreover, the mathematical formalism does not accept the massless toroidal voids. So, we have introduced an artificial medium, with very low density $20 \mathrm{~kg} \mathrm{~m}^{-3}$. In the following we will neglect the mass introduced by this medium but its material parameters will be important. In particular they are fitted in such a manner that the velocity of sound is small.

The applied numerical algorithm requires that the dimensions of the target area which is considered numerically must be much bigger than the size of an impactor. How much bigger it must be decided by the numerical experiments. Of course the greater energy of impactor the larger area must be considered. For high velocity impacts $\left(\approx 10 \mathrm{~km} \mathrm{~s}^{-1}\right)$ the considered dimensions of the target (radial times vertical) must be equal at least 10 radii of the impactor. In our case of an impactor with a radius $0.5 \mathrm{~m}$ the area of target showed in the figures is $8.5 \mathrm{~m}$ radially and $13.5 \mathrm{~m}$ in depth. However, the considered area in numerical calculation is twice greater. The total number of material points is $300 \times$ $200=60000$. The calculations have physical sens within a time interval which is no longer than that which is needed for reaching the borders of an area by the impact generated disturbances. This time is order of a ten milliseconds. This is certainly a time interval shorter than total time required for the crater formation. We estimated the last as being order of a time interval required for free fall of ejecta onto the nucleus surface. At the conditions of very low gravity field of a comet nucleus this time is of the order of an hour.

\section{EQUATIONS OF STATE (EOS) AND CONSTITUTIVE EQUATIONS (CE)}

In the following we consider the highly porous water icy target (comet nucleus) impacted by the meteoroid. Both are from materials of similar mean densities. The relation joining the mean density $\rho$, the porosity $\psi$, and the mineral content by mass (mass ratio of rock to rock plus ice) $C$, is as follow:

$\frac{1-\psi}{\rho}=\frac{1-C}{\rho_{i}}+\frac{C}{\rho_{r}}$

We adopt the following values of parameters $\psi=0.6, C=0.1$, and the densities of ice $\rho_{1}=940 \mathrm{~kg} \mathrm{~m}^{-3}$ and of rock $\rho_{\mathrm{r}}$ $=2400 \mathrm{~kg} \mathrm{~m}^{-3}$. Therefore, Eq. (1) gives a value of the mean density of the comet nucleus $\rho=400 \mathrm{~kg} \mathrm{~m}^{-3}$. The 2D axisymmetric hydrocode "HEFP" of free particles (Jach et al., 1994) used in this work contains the CE and the EOS as follow (according to Roddy et al., 1987):

$Y=\min \left(C_{o}+\alpha p, Y_{v m}\right)$,

$p=K_{m} x-\left(K_{m}-K_{0}\right) \mu^{*}\left[1-\exp \left(-x / \mu^{*}\right)\right]+\gamma \rho_{0}(1+x) E, \quad$ where $\quad x=\frac{\rho-\rho_{0}}{\rho_{0}}$

Here $p$ is the pressure, $E$ is the internal energy per unit mass, $Y$ is the yield strength of material in the Huber - von Mises sense, $C_{0}$ is the cohesion, $\alpha$ is the slope of the Mohr - Coulomb surface, and $Y_{\mathrm{vm}}$ is the von Mises limit for compression. Apart from the strength, the weak global gravity of the nucleus is also present in the calculations, see caption for Figure 4. For explanation of the other symbols see Table 1. Equations (2) and (3) are assumed to be valid for rock and for water ice. In this place it is worth to mention that, according to the authors knowledge, no EOS particularly valid for amorphous water ice is presently known. Therefore, our assumption that amorphous and crystalline ice are described by the same EOS is the most natural.

Our simulations require the numerical formalities appropriated for the modelling of continuous medium. Therefore the voids, dispersed in the icy-rocky target, must be simulated in a special manner. After several tests we have decide to model the voids as the area filled up with an artificial very low-dense medium. For this medium we have postulated the EOS is in the form of the Mie-Grüneisen equation:

$p=a x+b x^{2}+c x^{3}+\gamma \rho_{0}(1+x) E$. 
Here we have adopted the density $\rho_{0}=20 \mathrm{~kg} \mathrm{~m}^{-3}$ and the constants $a=1.91325 \times 10^{6} \mathrm{~Pa}, b=4.095 \times 10^{6} \mathrm{~Pa}, c=$ $7.9275 \times 10^{6} \mathrm{~Pa}$, and $\gamma=1.64$. Therefore, the velocity of sound is $\left[\left.(\mathrm{d} p / \mathrm{d} \rho)\right|_{\rho=\rho 0}\right]^{1 / 2}=\left(a / \rho_{0}+\gamma E\right)^{1 / 2}$ which is of the order of a few hundred meters per second. This is considerably smaller than this velocity in the real icy and rocky materials. The values of the parameters were chosen in such a way to accomplish the condition of low speed of sound in the artificial medium filling up the voids. This value is much less than the speeds of sound in the real media, rock and ice. Therefore the artificial medium does not play any role in transfer of disturbances through the real matter.

Table 1. Material constants of rock and of ice.

\begin{tabular}{|l|c|c|c|}
\hline \multicolumn{1}{|c|}{ Parameters of CE and of EOS } & Units & Rock & Ice \\
\hline$\rho_{0}-$ initial density & $\mathrm{kg} \mathrm{m}^{-3}$ & 2400 & 940 \\
\hline$K_{0}-$ initial bulk modulus & $10^{9} \mathrm{~Pa}$ & 24 & 2.2 \\
\hline$K_{\mathrm{m}}-$ maximum bulk modulus & $10^{9} \mathrm{~Pa}$ & 75 & 30 \\
\hline$C_{0}-$ cohesion coefficient & $10^{9} \mathrm{~Pa}$ & 0.5 & 0.5 \\
\hline$Y_{\mathrm{m}}-$ von Mises limit & $10^{9} \mathrm{~Pa}$ & 0.3 & 0 \\
\hline$\mu^{*}-$ exponential factor & - & 0.3 & 1.0 \\
\hline$\alpha-$ slope of Mohr-Coulomb surface & - & 0.75 & 0 \\
\hline$\gamma-$ Grïneisen coefficient & - & 1.3 & 1.6 \\
\hline
\end{tabular}

$\lg \mathrm{t}$

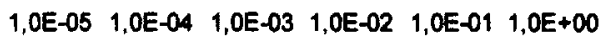

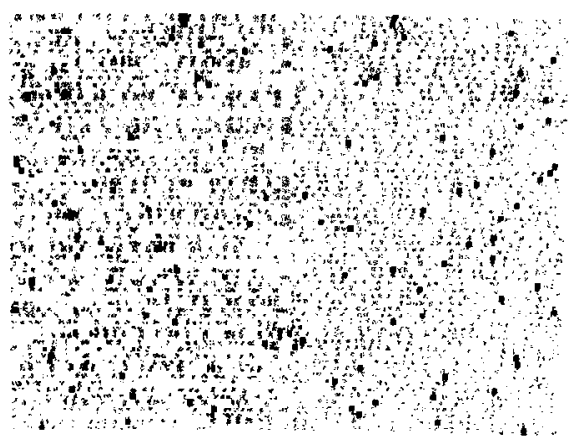

Fig. 1. Model of comet nucleus structure prior of an impact. Dark area - rock, shadowed area - ice, and empty spaces - voids. The diagram corresponds, in right proportions, to porosity $\psi=0.6$ and to rock concentration by mass $C=0.1$. Therefore ice occupies $38.3 \%$ by volume, rock $1.7 \%$ by volume and the rest are the voids.

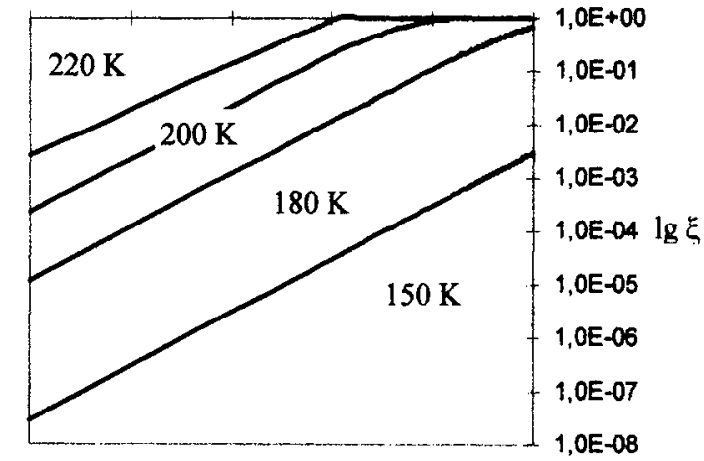

Fig. 2. Time dependence of amorphous $\Rightarrow$ crystalline phase transition function $\xi(t)$ according to eq. (5). Temperature $T$ is the parameter.

\section{RESULTS AND CONCLUSIONS}

The energy of impact is transformed into crater formation, into kinetic energy of ejecta, and into energy transferred by shock wave penetrating inward the impacted body. As a result the temperature of a layer of matter adjacent to the crater bowl increases. In certain areas it can exceed, for some time interval, the temperature appropriate for the fast transition from amorphous to crystalline state of water ice. This transition temperature can be estimated from the known function describing the kinetics of phase transition. The kinetics of the phase transition of water ice from 
amorphous form to crystalline ice follows the relation (Haruyama et al., 1993, Jenniskens and Blake, 1994, Kouchi et al., 1994):
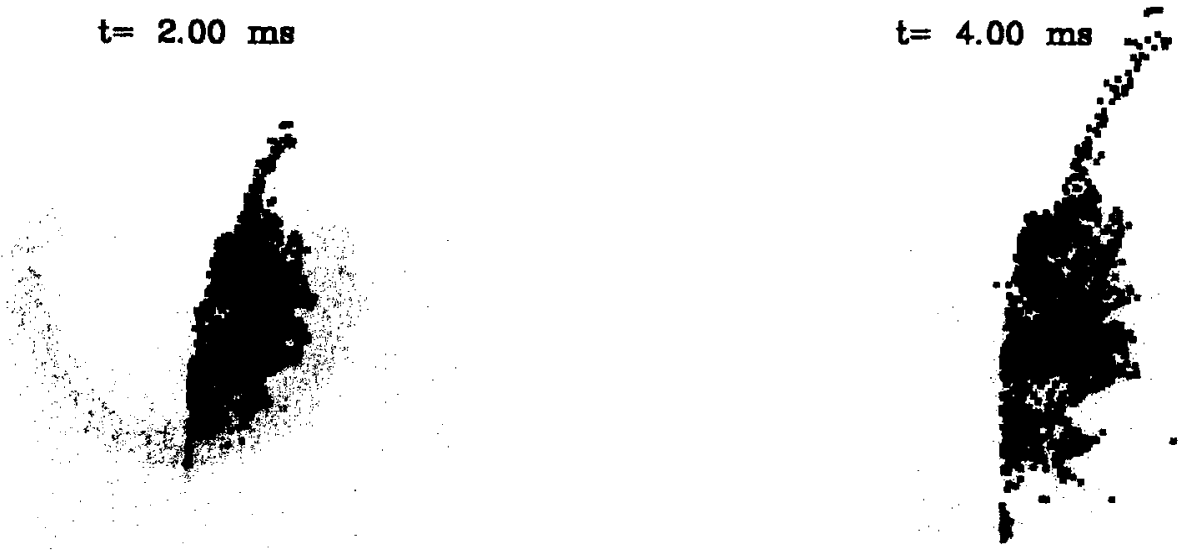

Fig. 3. Distributions of the temperature as the results of the simulation of a normal impact on a comet nucleus: (a) at time $2 \mathrm{~ms}$ after impact, and (b) after $4 \mathrm{~ms}$. Impactor radius is $0.5 \mathrm{~m}$ and its velocity is $10 \mathrm{~km} \mathrm{~s}^{-1}$. Target area in the figure is $17 \mathrm{~m}$ horizontally and $13.5 \mathrm{~m}$ in depth. The initial temperature was $50 \mathrm{~K}$ and it remains unchanged far away from an impact site. In the shocked zone the temperature seriously increases. In the area of the ejecta outflow the temperature decreases. The problem is axisymmetric and therefore the figure should be axisymmetric too. However, to present more information, the diagram is divided onto left and right hand sides. On the right hand side of the both diagrams the points in which the temperature has reached $200 \mathrm{~K}$ (at any moment from the beginning to the actual time) are marked in black. At these points one can expect that the transformation from the amorphous to the crystalline phase was considerably advanced, see Figure 2 . The gray graduation from light to dark relates to the actual temperature from cooler to warmer, respectively.

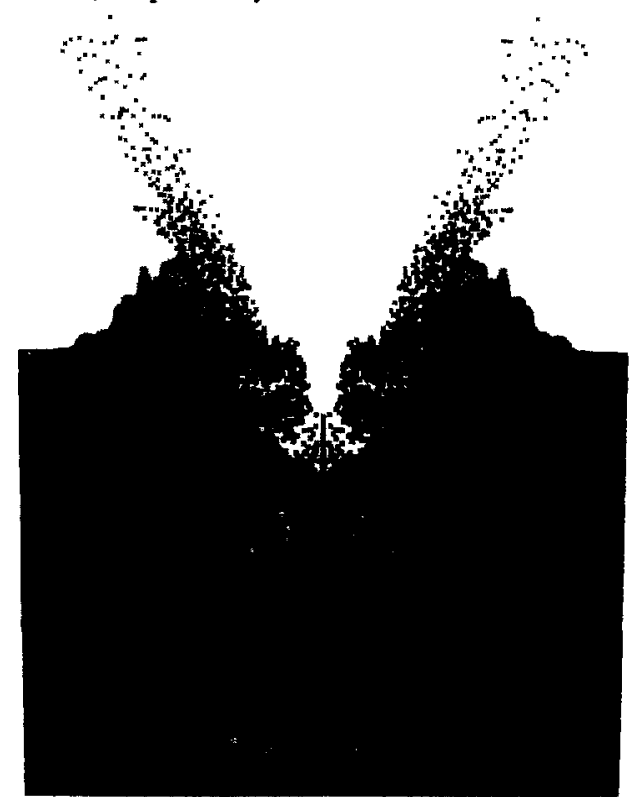

Fig. 4. The instantaneous shape of the crater formed on comet nucleus surface $4 \mathrm{~ms}$ after an impact. Light gray area presents the matter escaping from the comet, since its velocity perpendicular to the surface is larger then $0.3 \mathrm{~m} \mathrm{~s}^{-1}$. Black area illustrates a medium that will remain on the comet. Note the retreat of comet surface about $6 \mathrm{~m}$ below the initial level. The white bow corresponds to an actual position of the shock front. 
Here $\xi$ is the factor of amorphous ice already transformed to crystalline form during time interval $t$ seconds at temperature $\mathrm{T}$ kelvins. This equation leads to $\xi$ reaching the value equal to about 0.5 during the time interval of the order of 10 milliseconds for the temperature being some kelvins above the value $200 \mathrm{~K}$. By the way, the temperature $135 \mathrm{~K}$ which relates to experiments performed in the laboratories requires time interval of the order of hours to reach $\xi=0.5$, see Figure 2 . In the right hand side of the Figure 3 the points that have reached the temperature $200 \mathrm{~K}$ at least, in any moment after the impact, are marked in black. The results are illustrated in Figure 3 on the right hand side of this figure. One can find that in our case of impact velocity equal to $10 \mathrm{~km} \mathrm{~s}^{-1}$ the area in which the temperature $T \geq T_{\mathrm{a}}$ has been reached is considerably larger than the impactor size. Therefore, meteoritic impact can transform the comet subsurface ice, possibly amorphous, to the crystalline state. However, the calculations performed with impact velocity of the order of $1 \mathrm{~km} \mathrm{~s}^{-1}$ or lower show that transformation is not possible. The impactor density in our calculations was only $400 \mathrm{~kg} \mathrm{~m}^{-3}$. For higher density of the impactors the velocities sufficient to involve phase transition can be considerably smaller. Figure 4 presents the late state of crater formation. This is close to the final shape of the crater.

The conclusions can be divided for two almost independent groups. They are:

(1) Conclusions concerning the physics of impact:

(a) Modelling of low dense and multicomponent comet nucleus as layered target with voids seems to be reasonable. In future, the calculations with improved EOS for different media are planed. The studies related to the large intervals of parameters (impact velocity, impactor size) are also required.

(b) Meteoritic impacts can induce amorphous to crystalline transition in cometary water ice.

(2) Conclusions related to the applied algorithm:

(a) Artificial low dense medium filling the voids allows to apply the methods of the mechanics of continuum.

(b) Our method, as we tested, can be applied also for low velocity impacts, and therefore we can consider the impact of the Rosetta lander.

\section{ACKNOWLEDGMENTS}

This work was partially supported by grants 6 P04D 00910 and 9 T12B 00114 from the KBN (Poland).

\section{REFERENCES}

Haruyama, J., T. Yamamoto, H. Mizutani, and J. M. Grynberg, Thermal history of comets during residence in the Oort cloud: effect of radiogenic heating in combination with the very low thermal conductivity of amorphous ice, J. Geophys. Res., 98, E8, 15079-15090 (1993).

Jach, K., J. Leliwa-Kopystyński, M. Mroczkowski, R. Świerczyński and P. Wolański, Free particle modelling of hypervelocity asteroid collisions with the Earth, Planet. Space Sci., 42, 1123-1137 (1994).

Jenniskens, P., and D. F. Blake, Structural transitions in amorphous water ice and astrophysical implications, Science, 265, 753-756, (1994).

Kouchi, A., T. Yamamoto, T. Kozasa, T. Kuroda, and J. M. Greenberg, Conditions for condensation and preservation of amorphous ice and crystallinity of astrophysical ices, Astron. Astrophys, 290, 1009-1018 (1994).

Podolak, M., and D. Prialnik, Models of the structure and evolution of comet P/Wirtanen, Planet. Space Sci., 655664 (1996).

Prialnik, D., and A. Bar-Nun, Radiogenic heating of comets by $\mathrm{Al}^{26}$ and implications for their time of formation, Astrophys. J., 319, 993-1002 (1987).

Rickman, H., The thermal history and structure of cometary nuclei, in Comets in the post-Halley era, Vol. 2, pp. 733-760, eds. R. L. Newburn, Jr., M. Neugebauer, and J. Rahe. Kluwer Acad. Publ. (1991).

Roddy, D. J., S. H. Schuster, M. Rosenblatt, L. B. Grant, P. J. Hassing, and K. N. Kreyenhagen, Computer simulations of large asteroid impacts onto oceanic and continental sites preliminary results on atmospheric, cratering and ejecta dynamics, Int. J. Impact Engng., 5, 525-541 (1987). 